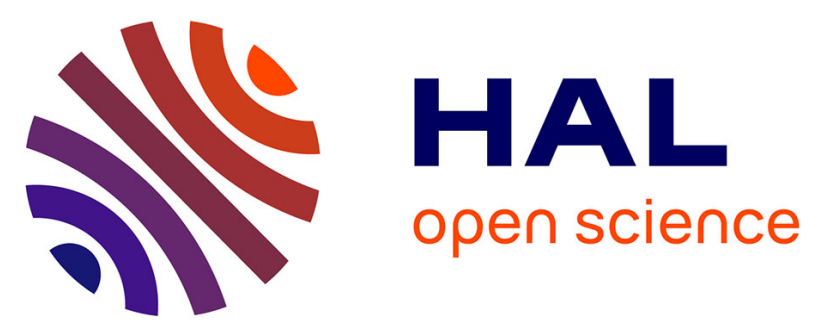

\title{
Quantitative dSTORM super-resolution microscopy localizes Aurora kinase A/AURKA in the mitochondrial matrix
}

\author{
Béatrice Durel, Charles Kervrann, Giulia Bertolin
}

\section{- To cite this version:}

Béatrice Durel, Charles Kervrann, Giulia Bertolin. Quantitative dSTORM super-resolution microscopy localizes Aurora kinase A/AURKA in the mitochondrial matrix. Biology of the Cell, 2021, 113 (11), pp.458-473. 10.1111/boc.202100021 . hal-03367309

\author{
HAL Id: hal-03367309 \\ https://hal.science/hal-03367309
}

Submitted on 18 Oct 2021

HAL is a multi-disciplinary open access archive for the deposit and dissemination of scientific research documents, whether they are published or not. The documents may come from teaching and research institutions in France or abroad, or from public or private research centers.
L'archive ouverte pluridisciplinaire HAL, est destinée au dépôt et à la diffusion de documents scientifiques de niveau recherche, publiés ou non, émanant des établissements d'enseignement et de recherche français ou étrangers, des laboratoires publics ou privés.

\section{(ㅇ)(1) $\$$}

Distributed under a Creative Commons Attribution - NonCommercial - NoDerivatives| 4.0 


\title{
Quantitative dSTORM super-resolution microscopy localizes Aurora kinase A/AURKA in the mitochondrial matrix
}

\author{
Béatrice Durel ${ }^{1} \quad$ Charles Kervrann ${ }^{2,3} \quad$ Giulia Bertolin ${ }^{4}$ (1)
}

${ }^{1}$ Cell Imaging Platform, Structure Fédérative de Recherche Necker, INSERM US24, CNRS UMS3633, Paris F-75015, France

${ }^{2}$ Serpico Project-Team, Inria - Centre Inria Rennes-Bretagne Atlantique, CNRS UMR144, Campus Universitaire de Beaulieu, Rennes F-35042, France

${ }^{3}$ Institut Curie, PSL Research University, Paris F-75005, France

${ }^{4}$ CNRS, Univ Rennes, IGDR (Institute of Genetics and Development of Rennes), UMR 6290, Rennes F-35000, France

\section{Correspondence}

Giulia Bertolin, CNRS, Univ Rennes, IGDR

(Institute of Genetics and Development of

Rennes), Rennes, France.

Email:giulia.bertolin@univ-rennes1.fr

Funding information

Fondation ARC pour la Recherche sur le Cancer; Réseau Technologique Microscopie photonique de Fluorescence Multidimensionnelle; Ligue Contre le Cancer; France Biolmaging

\begin{abstract}
Background information: Mitochondria are dynamic organelles playing essential metabolic and signaling functions in cells. Their ultrastructure has largely been investigated with electron microscopy (EM) techniques. However, quantifying protein-protein proximities using EM is extremely challenging. Superresolution microscopy techniques as direct stochastic optical reconstruction microscopy (dSTORM) now provide a fluorescent-based, quantitative alternative to EM. Recently, super-resolution microscopy approaches including dSTORM led to valuable advances in our knowledge of mitochondrial ultrastructure, and in linking it with new insights in organelle functions. Nevertheless, dSTORM is mostly used to image integral mitochondrial proteins, and there is little or no information on proteins transiently present at this compartment. The cancer-related Aurora kinase A/AURKA is a protein localized at various subcellular locations, including mitochondria.

Results: We first demonstrate that dSTORM coupled to GcoPS can resolve protein proximities within individual submitochondrial compartments. Then, we show that dSTORM provides sufficient spatial resolution to visualize and quantify the most abundant pool of endogenous AURKA in the mitochondrial matrix, as previously shown for overexpressed AURKA. In addition, we uncover a smaller pool of AURKA localized at the OMM, which could have a potential functional readout. We conclude by demonstrating that aldehyde-based fixatives are more specific for the OMM pool of the kinase instead.

Conclusions: Our results indicate that dSTORM coupled to GcoPS colocalization analysis is a suitable approach to explore the compartmentalization of non-integral mitochondrial proteins as AURKA, in a qualitative and quantitative manner. This method also opens up the possibility of analyzing the proximity between AURKA and its multiple mitochondrial partners with exquisite spatial resolution, thereby allowing novel insights into the mitochondrial functions controlled by AURKA.

Significance: Probing and quantifying the presence of endogenous AURKA a cell cycle-related protein localized at mitochondria - in the different organelle subcompartments, using quantitative dSTORM super-resolution microscopy.
\end{abstract}

KEYWORDS

AURKA, colocalization, GcoPS, mitochondria, super-resolution, two-color dSTORM

This is an open access article under the terms of the Creative Commons Attribution-NonCommercial-NoDerivs License, which permits use and distribution in any medium, provided the original work is properly cited, the use is non-commercial and no modifications or adaptations are made.

() 2021 The Authors. Biology of the Cell published by Wiley-VCH GmbH on behalf of Société Française des Microscopies and Société de Biologie Cellulaire de France 


\section{INTRODUCTION}

Mitochondria are multifunctional organelles involved in a wide range of cellular functions and signaling pathways. They are organized in an interconnected network spread throughout the cell, and in contact with several other subcellular compartments (van der Laan et al., 2016). Mitochondria are composed of two membranes: the Outer Mitochondrial Membrane (OMM), which encloses the organelle, and the Inner Mitochondrial Membrane (IMM), which is folded in invaginations called cristae and contains the oxidative phosphorylation system (OXPHOS). Two soluble compartments are also present: the Intermembrane Space (IMS), which separates the OMM from the IMM, and the matrix, which is the innermost compartment and where mtDNA molecules are located (Nunnari \& Suomalainen, 2012).

In the past decades, our understanding of mitochondrial sub-architecture greatly benefited from advances in electron microscopy (EM). This technique turned out to be particularly useful in revealing how the two membranes are organized in various cell types and in tissues (reviewed in (Frey \& Mannella, 2000)), and in highlighting how mitochondria are structurally altered in disease (Vincent et al., 2016; Siegmund et al., 2018). However, EM is less convenient to explore the intramitochondrial localization of individual proteins. To be detected, antibodies specific to the protein of interest need to be coupled to gold beads-an approach known as immunogold EM. Although this allows to visualize the sub-mitochondrial distribution of a protein with exquisite spatial resolution (Vogel et al., 2006), immunogold EM is a procedure with a relatively low efficiency. In individual EM sections, gold particles are generally scarce, and it is therefore nearly impossible to conclude on the localization of a protein by looking at single mitochondria. To extract quantitative information, individual localizations must be calculated from a considerable number of images before obtaining an average localization coefficient (Enger, 2017; Hayat, 1992). To explore protein associations in cells, double-immunogold EM is particularly convenient (Boykins et al., 2016). This consists of imaging two proteins within the same sample using gold beads of different sizes. With such approach, beads of 10 and $20 \mathrm{~nm}$ were used to detect the colocalization of mitochondrial complex III and IV in individual mitochondria (Golic et al., 2016). However, images of mitochondria with two sizes of gold beads are generally hard to interpret and to quantify, especially when beads are adjacent or clustered together.

Recent advances in fluorescence microscopy offer elegant and more quantitative alternatives to electron microscopy. The width of a mitochondrion is between 200 and $500 \mathrm{~nm}$, and the two mitochondrial membranes are 10-20 nm apart (Kaasik et al., 2007). Therefore, these organelles are beyond the resolution limit of most optical microscopes. This limit makes conventional fluorescence microscopy not suitable to explore the distribution of proteins within specific submitochondrial compartments. The recent development of super-resolution techniques allows to go beyond this diffraction limit, and it is now easier to investigate mitochondrial protein distribution in a quantitative manner (Jakobs \& Wurm, 2014). Among super-resolution approaches, direct stochastic optical reconstruction microscopy (dSTORM) uses the transitions between the on- and off-states of fluorophores for multiple imaging cycles, which result in the activation/deactivation of fluorophores in a stochastic manner (Heilemann et al., 2008), (reviewed in (Samanta et al., 2019)). A high-resolution map of individual fluorophores is obtained by recording the activation/deactivation rates of each fluorophore and their respective $x y z$ coordinates. Given that this method provides an optical resolution of $\sim 10 \mathrm{~nm}$, well beyond the diffraction limit, dSTORM immediately showcased its potential for mitochondrial imaging. It was used to image mitochondria-microtubule contacts (Huang et al., 2008), the distribution of specific proteins as the ATPsynthase $\mathrm{F} 1 \propto$ subunit or the MICOS complex (Dlasková et al., 2018; Stephan et al., 2020), and to explore the localization of mitochondrial nucleoids (Dlasková et al., 2018).

Integral mitochondrial proteins such as MICOS subunits showed very little or no extra-mitochondrial signal in previous dSTORM analyses (Stephan et al., 2020). When a protein is known to localize exclusively at mitochondria, the signal outside the organelles can be easily interpreted and treated as an aspecific, background noise. However, discriminating between real and aspecific signals is a greater challenge when a given protein has both a mitochondrial and an extra-mitochondrial localization. This is the case of the Ser/Thr kinase AURKA, which has multiple subcellular locations including the centrosome and the mitotic spindle (reviewed in (Nikonova et al., 2013)), the nucleus (reviewed in (Naso et al., 2021)), and mitochondria (Bertolin et al., 2018; Grant et al., 2018). AURKA is frequently overexpressed in epithelial tumors, making it a target of primary interest for the development of anti-cancer therapies (Damodaran et al., 2017). At mitochondria, AURKA was shown to regulate organelle dynamics and ATP levels throughout the cell cycle (Bertolin et al., 2018; Grant et al., 2018; Kashatus et al., 2011). Although it is conceivable that the regulation of these functions requires the interaction of AURKA with a great number of mitochondrial partners, we still have a partial view of the mitochondrial signaling cascade(s) in which AURKA is involved. Determining protein/protein proximities between AURKA and its interactors with an increased resolution at the submitochondrial level would significantly increase our understanding of how AURKA orchestrates its multiple functions at this compartment. 
Our first goal was to evaluate the sensitivity of the twocolor dSTORM approach in quantitatively separating the OMM and IMM compartments, as well as integral mitochondrial proteins within the OMM or the IMM/matrix subcompartments. We then used this method to determine the submitochondrial distribution of AURKA, and we optimized fixation procedures to limit the contribution of the extra-mitochondrial pool of the kinase. With this strategy, we uncovered the existence of two pools of AURKA with a distinct submitochondrial localization and with a potential functional relevance.

\section{RESULTS}

\section{Separating the OMM and IMM using integral mitochondrial markers and quantitative dSTORM analyses}

We first aimed at exploring whether dSTORM was a suitable approach to spatially distinguish the OMM from the IMM in MCF7 cells. To this end, we selected integral proteins with a known OMM or IMM localization, thereby allowing us to distinguish the two compartments unambiguously. MCF7 cells were chosen as their mitochondrial network is naturally fragmented (Daniel et al., 2019; Sarmiento-Salinas et al., 2019), and subcompartments could readily be ascribed to individual organelles. We first fixed the cells with a mixture of formaldehyde/glutaraldehyde (PFA/G) to maintain mitochondrial morphology. We then labeled the OMM with an anti-TOMM22 primary antibody and we used it in combination with a primary antibody targeting the IMM marker COX2, or another targeting the IMM/matrix marker PMPCB. Two-color dSTORM recordings in 2D successfully managed to image TOMM22 together with COX2 (Figure 1(a)), and with PMPCB (Figure 1(c)). The TOMM22-specific staining appeared as a noncontiguous, ring-like structure enclosing individual mitochondria, while COX2 and PMPCB labeled mitochondria on the inside and they were thoroughly distributed throughout the mitochondrial surface (Figure 1(a-c)).

Given that OMM and IMM/matrix markers qualitatively showed a certain degree of superposition, we quantified it by preforming colocalization analyses. We used GcoPS, a hybrid object-based and intensity-based colocalization method which is rapid, robust in treating noise, able to handle complex image patterns, and with a comparable precision both on whole images and on image subregions (Lavancier et al., 2019). At mitochondria, the colocalization coefficient for the TOMM22/COX2 and the TOMM22/PMPCB pairs was $\sim 30 \%$ (Figure $1(\mathrm{e})$ ). Within individual mitochondria, GcoPS also detected areas where OMM and IMM/matrix proteins show anticolocalization, which is the probability that two proteins are not in contact with each other (Figure 1(b-d), left pan- els) (Bolte \& Cordelieres, 2006). In support to this observation, colocalization heatmaps show that the two protein pairs do not colocalize throughout the two mitochondrial membranes, but colocalization is rather concentrated in discrete proximity sites between the OMM and the IMM/matrix (Figure $1(b-d)$, middle panels). These sites are reminiscent of contact sites between the OMM and the IMM, as described in previous reports using EMbased approaches (Reichert \& Neupert, 2002).

Using integral mitochondrial proteins as markers, our results show that dSTORM and GcoPS are capable to separate the OMM from the IMM/matrix. Furthermore, our approach is also capable of visualizing discrete sites of colocalization between the two compartments, which are reminiscent of membrane contact sites.

\section{Establishing protein colocalization on individual mitochondrial membranes}

Given that dSTORM can globally separate the outer from the inner mitochondrial compartment, we then sought to determine its power in determining protein colocalization within the same mitochondrial subcompartment. On the OMM, we explored the colocalization of TOMM22 with either VDAC1 or TOMM20. TOMM20 is another member of the Translocase of Outer Membrane (TOMM) complex, and a physical interactor of TOMM22 (van Wilpe et al., 1999). On the contrary, VDAC1 does not belong to the TOMM complex (Camara et al., 2017), and it is expected to display a lower degree of colocalization with TOMM22. While TOMM22 was distributed throughout the OMM, we observed that VDAC1 is not uniformly distributed throughout the OMM, but it rather clusters in TOMM-positive distinct domains (Figure 2(a)). This confirms previous reports obtained with STED microscopy in U2OS cells (Neumann et al., 2010). GcoPS analyses revealed that the colocalization degree of VDAC1 with TOMM22 was partial, and the median colocalization values did not exceed 25\% (Figure 2(a,b,e)). Conversely, the colocalization degree between the two TOMM subunits TOMM22 and TOMM20 was significantly higher, and it reached $60 \%$ for the majority of the cells analyzed (Figure 2(c-e)). These results indicate that dSTORM microscopy coupled to GcoPS analyses is capable of separating integral proteins localized within the OMM.

Following a similar strategy, we explored whether dSTORM and GcoPS analyses can determine protein proximities at the $\mathrm{IMM} /$ matrix. To this end, we calculated the degree of colocalization between the IMM protein ATP5B, and COX2, or between ATP5B and PMPCB. Given that ATP5B is an integral IMM protein facing the matrix compartment (Bozgeyik et al., 2015), we expected it to show a higher degree of colocalization with PMPCB than with COX2. First, we observed that ATP5B, COX2 and PMPCB are uniformly distributed 
a
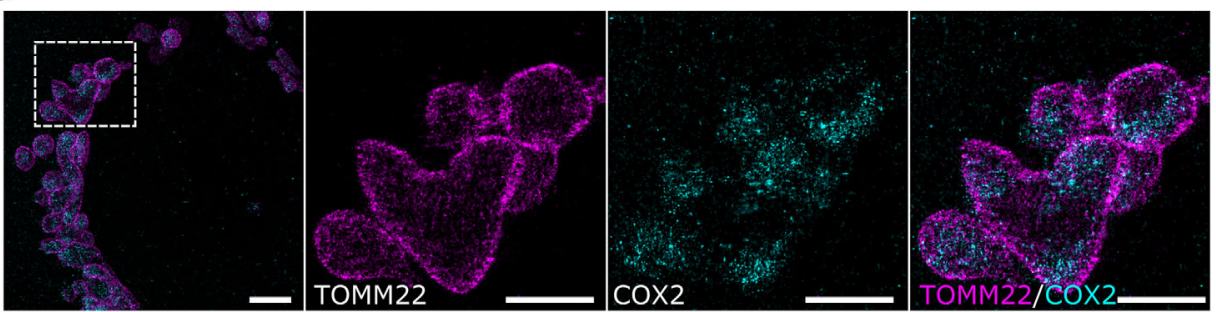

b

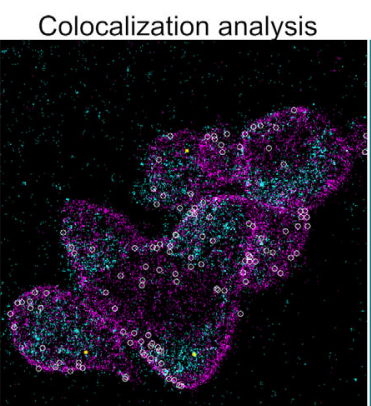

Colocalization heatmap

Colocalization windows
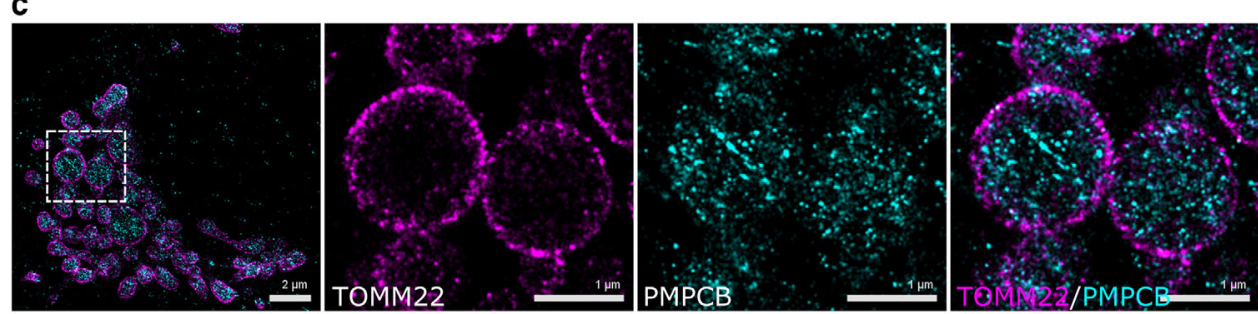

d Colocalization analysis Colocalization heatmap Colocalization windows
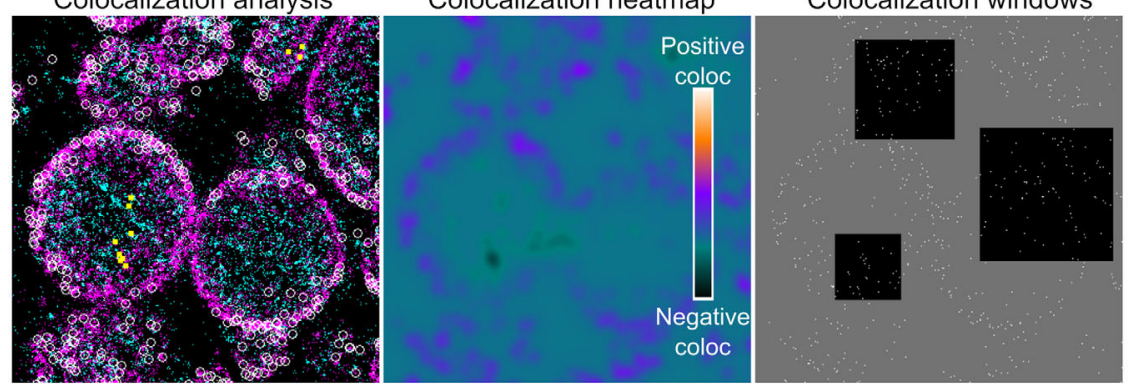

E

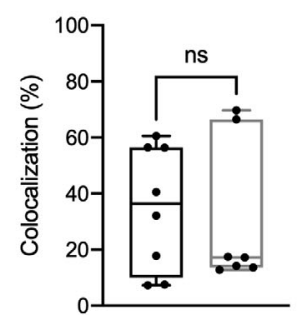

TOMM22/COX2

TOMM22/PMPCB

FIGURE 1 The OMM marker TOMM22 is separated from the IMM marker COX2 and the matrix protein PMPCB. (a-c) Maximal projections of representative 2D dSTORM micrographs from MCF7 co-stained for TOMM22 and COX2 (a), or for TOMM22 and PMPCB (c). The anti-TOMM22 primary antibody was detected using a secondary antibody conjugated to Alexa 647 and pseudocolored magenta, while anti-COX2 and anti-PMPCB primary antibodies were detected using secondary antibodies conjugated to Alexa 555 and pseudocolored cyan. The dotted area in the left panels indicates the magnified region where each staining is shown individually (middle panels), or merged (right panels). Scale bar: $2 \mu \mathrm{m}$ (left) or $1 \mu \mathrm{m}$ (middle and right panels). (b-d) (Left panels) Visual representation of positive (white rounds) and negative (yellow squares) colocalization sites on the magnified area of TOMM22/COX2 (b) and of TOMM22/PMPCB (d) micrographs after GcoPS analysis. (Middle panels) Colocalization heatmap ranging from yellow (positive colocalization) to black (negative colocalization), and background (colocalization score arbitrarily set to 0 ) in green. (Right panels). Three representative colocalization windows of $64 \times 64$ pixels, $96 \times 96$ pixels and $128 \times 128$ pixels superimposed over 3 representative positions. For each position, a window center is drawn in the set of super-localizations of the reference channel, and labeled as a white spot which is used for colocalization. All the window centers tested are shown. Image size: $4922 \times 4981$ in (b), $1500 \times 1500$ in (d). (e) Quantification of the percentage of colocalization for the indicated protein pairs. Data range from min to max. Dots correspond to individual cells issued from $n=3$ independent experiments. ns, not significant 


\section{a}

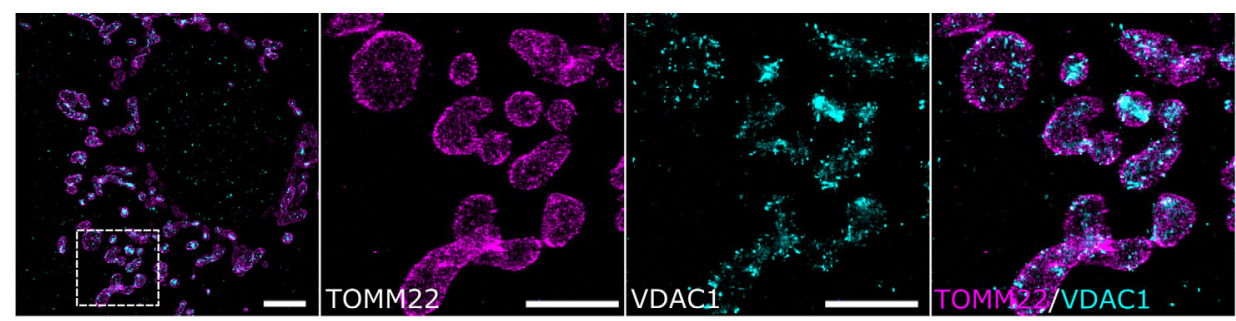

b

Colocalization analysis Colocalization heatmap

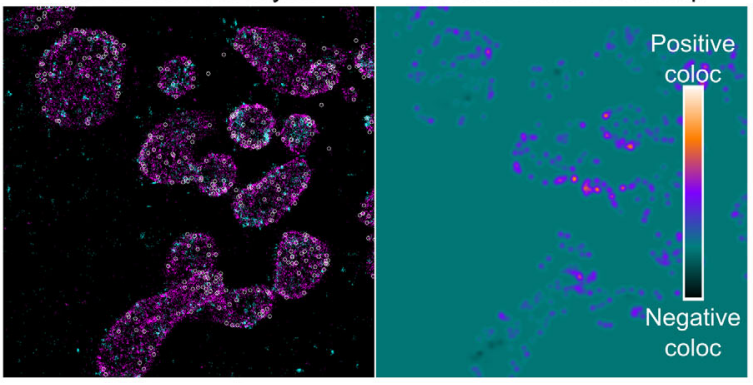

C
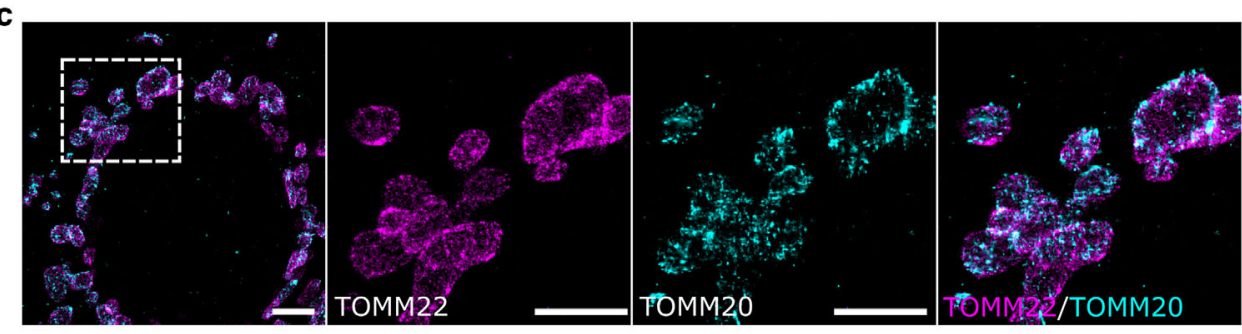

d

Colocalization analysis

Colocalization heatmap
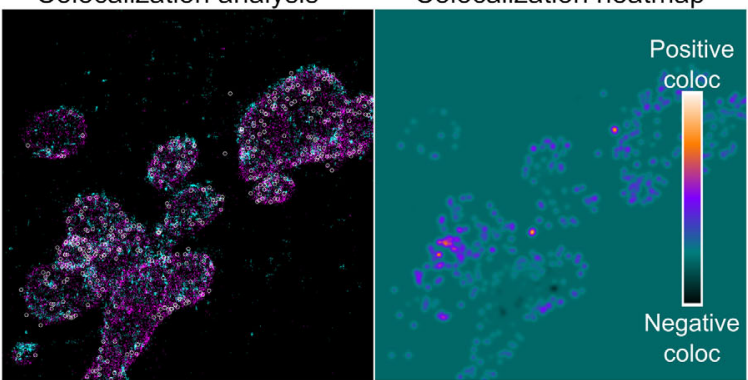

e

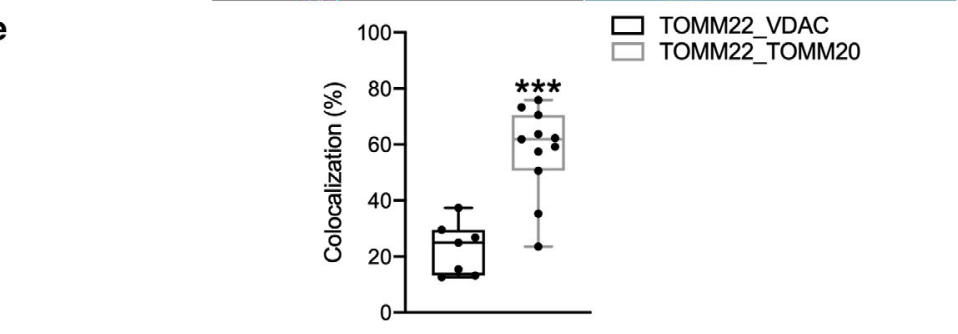

TOMM22 VDAC

TOMM22_TOMM20

FIGURE 2 The OMM marker TOMM22 preferentially colocalizes with TOMM20 on the OMM. (a-c) Maximal projections of representative $2 \mathrm{D}$ dSTORM micrographs from MCF7 co-stained for TOMM22 and VDAC1 (a), or for TOMM22 and TOMM20 (c). The anti-TOMM22 primary antibody was detected using a secondary antibody conjugated to Alexa 647 and pseudocolored magenta, while anti-VDAC1 and anti-TOMM20 primary antibodies were detected using secondary antibodies conjugated to Alexa 555 and pseudocolored cyan. The dotted area in the left panels indicates the magnified region where each staining is shown individually (middle panels), or merged (right panels). Scale bar: $2 \mu \mathrm{m}$ (left) or $1 \mu \mathrm{m}$ (middle and right panels). (b-d) (Left panels) Visual representation of positive (white rounds) colocalization sites on the magnified area of TOMM22/VDAC1 (b) and of TOMM22/TOMM20 (d) micrographs after GcoPS analysis. (Right panels) Colocalization heatmap ranging from yellow (positive colocalization) to black (negative colocalization), and background (colocalization score arbitrarily set to 0 ) in green. Image size: $6003 \times 6040$ in (b), $5993 \times 6062$ in (d). (e) Quantification of the percentage of colocalization for the indicated protein pairs. Data range from min to max. Dots correspond to individual cells issued from $n=3$ independent experiments. ${ }^{* * *} p<0.001$ 
on the IMM/matrix (Figure 3(a,c)). Then, GcoPS analyses determined a median colocalization value greater than $40 \%$ for both pairs analyzed, which suggests that the proteins are globally located in proximity. As expected, however, the colocalization between ATP5B and PMPCB was significantly higher than the one calculated between ATP5B and COX2 (Figure 3(b,d,e)). This is consistent with the relative positioning of ATP5B on the matrix-facing side of the IMM, while COX2 is embedded within the IMM and does not face the matrix.

Overall, these data highlight the power of dSTORM coupled to GcoPS analyses to detect protein proximities within individual mitochondrial subcompartments, such as the OMM or the IMM/matrix.

\section{dSTORM in methanol-fixed cells uncovers a larger fraction of endogenous AURKA in the mitochondrial matrix, and a smaller fraction on the OMM}

After establishing that dSTORM is a suitable approach to localize mitochondrial integral proteins with known localization, we explored whether this technique could be used to monitor the presence of non-integral mitochondrial proteins and their relative submitochondrial distribution. We used the cell cycle-related kinase AURKA as an example of protein localizing in multiple cellular compartments at a time, including mitochondria. Previous approaches combining the overexpression of AURKA and EM-based techniques localized the kinase in the mitochondrial matrix. (Bertolin et al., 2018). However, we reasoned that endogenous and overexpressed AURKA could differentially localize within mitochondria. Therefore, we explored the submitochondrial localization of endogenous AURKA by testing the colocalization of the kinase with key mitochondrial components.

To limit the contribution of the cytosolic, extramitochondrial signal, we first fixed MCF7 cells with methanol. Methanol, as other similar alcohol-based fixatives, is known to extract cytosolic and nuclear proteins (Hoetelmans et al., 2001; Schnell et al., 2012). We then co-stained the cells with AURKA and TOMM20 to label the OMM, or PMPCB to label the IMM/matrix (Figure 4(a,c)). GcoPS-based analyses revealed that the colocalization between AURKA and TOMM20 does not exceed $17 \%$ (Figure 4(b,e)), while the one between AURKA and PMPCB is two-fold higher and nearly reaches 38\% (Figure 4(d,e)).

Our data indicate that dSTORM analyses performed on methanol-fixed cells localize endogenous AURKA mainly in the mitochondrial matrix. In this light, they confirm previous EM data performed with overexpressed AURKA (Bertolin et al., 2018). While the larger pool of the kinase is located in the matrix in ISTORM analyses, we also retrieved a smaller pool of AURKA local- ized on the OMM and colocalizing with the TOMM20 marker. The two pools are not mutually exclusive, as AURKA needs to be imported through the OMM before reaching the matrix, and it was shown to interact with the TOMM complex itself and with other OMM proteins (Bertolin et al., 2018). For the first time, our results allow to visualize these two pools of endogenous AURKA, one at the $\mathrm{OMM}$ and one in the mitochondrial matrix.

\section{PFA/G fixation coupled to antigen retrieval procedures is more specific for OMM-localized AURKA}

Despite the usefulness of methanol to limit the contribution of the extra-mitochondrial signal of AURKA, we reasoned that it also induced a partial loss of mitochondrial protein content due to its lipid-dissolving action (Hoetelmans et al., 2001; Jamur \& Oliver, 2010; Melan, 1999). Therefore, we compared the performance of methanol- and PFA/G-based fixation methods to preserve mitochondrial integrity. The distribution of TOMM20 was similar to the one of TOMM22 in PFA/Gfixed cells, with a well-defined ring-like structure and a non-contiguous staining (Figure 5(a), compare with Figure $1(\mathrm{a})$ ). On the contrary, the OMM appeared ruptured, and with an irregular TOMM20 staining in methanolfixed cells. A similar loss in mitochondrial content was also observed for PMPCB, which appeared fragmented and not contiguous upon fixation with methanol, while the matrix appeared intact when cells were fixed with PFA/G (Figure 5(b)).

As shown above, dSTORM coupled to GcoPS determined that endogenous AURKA mainly colocalizes with the matrix marker PMPCB in methanol-fixed MCF7 cells. However, alcohol-based fixation methods as methanol may alter the visualization of protein proximities, due to the partial loss in mitochondrial protein content they induce (Figure 5). In this light, we sought to improve sample preparation procedures to preserve mitochondrial ultrastructure while visualizing the kinase at this location. Therefore, we first fixed cells with PFA/G to maintain mitochondrial integrity. Then, we used antigen retrieval methods to expose the epitope of AURKA recognized by the anti-AURKA primary antibody, which is normally hidden by PFA/G (Cremet et al., 2003). As in methanol-fixed cells, we then co-stained cells with AURKA and TOMM20 to label the OMM, or PMPCB to label the matrix. We first observed that mitochondria stained for TOMM20 or for PMPCB were also positive for AURKA, thereby indicating that antigen retrieval procedures were successful in detecting the kinase under PFA/G fixation conditions (Figure 6(a,c)). We then used GcoPS-based analyses to determine the degree of colocalization between AURKA and TOMM20 or PMPCB. The colocalization coefficients of the AURKA/TOMM20 


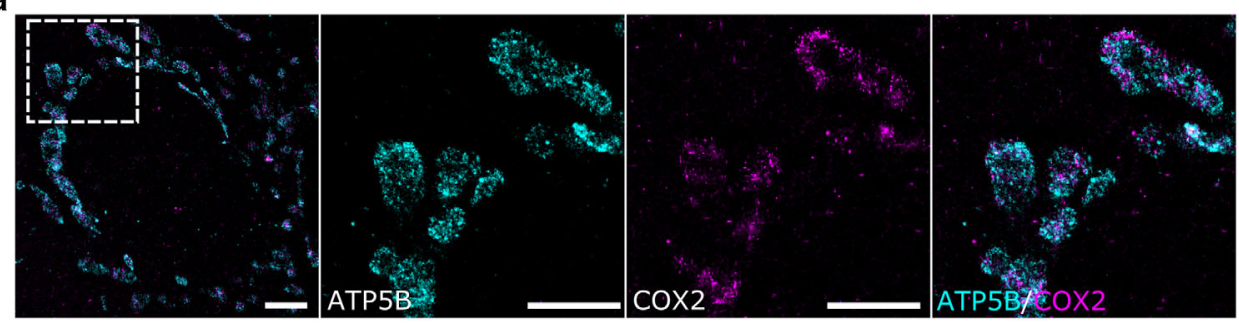

b

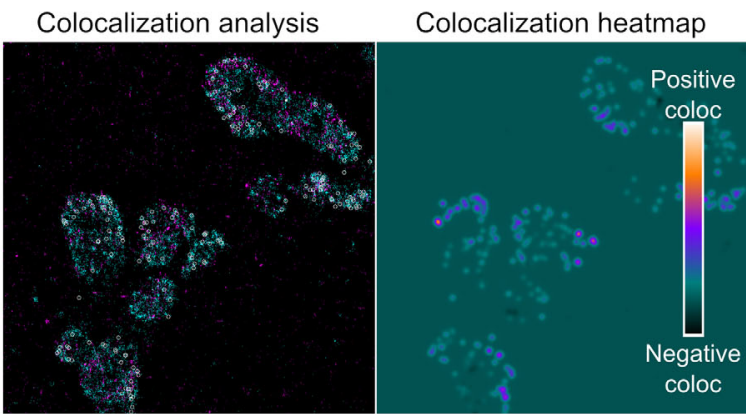

C

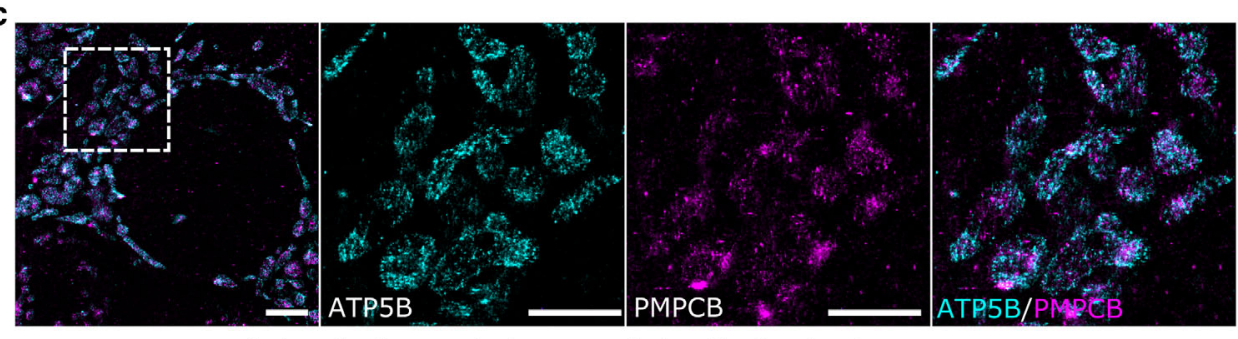

d

Colocalization analysis

Colocalization heatmap
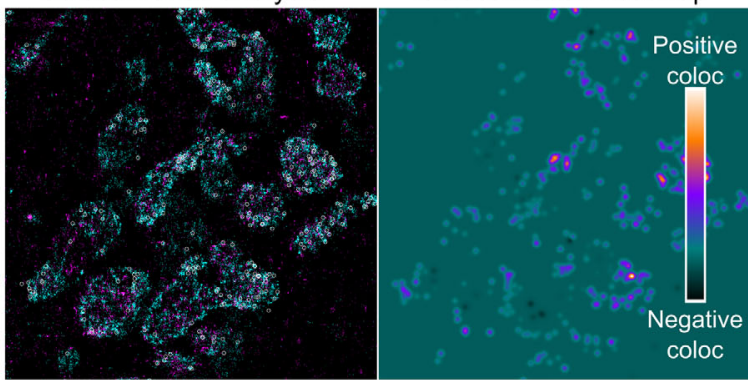

e

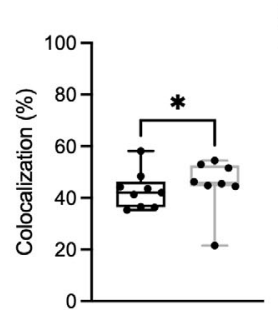

$\square$ ATP5B_COX2

FIGURE 3 The IMM/matrix marker ATP5B preferentially colocalizes with PMPCB. (a-c) Maximal projections of representative 2D dSTORM micrographs from MCF7 co-stained for ATP5B and COX2 (a), or for ATP5B and PMPCB (c). The anti-ATP5B primary antibody was detected using a secondary antibody conjugated to Alexa 647 and pseudocolored cyan, while anti-COX2 and anti-PMPCB primary antibodies were detected using secondary antibodies conjugated to Alexa 555 and pseudocolored magenta. The dotted area in the left panels indicates the magnified region where each staining is shown individually (middle panels), or merged (right panels). Scale bar: $2 \mu \mathrm{m}$ (left) or $1 \mu \mathrm{m}$ (middle and right panels). (b-d) (Left panels) Visual representation of positive (white rounds) colocalization sites on the magnified area of ATP5B/COX2 (b) and of ATP5B/PMPCB (d) micrographs after GcoPS analysis. (Right panels) Colocalization heatmap ranging from yellow (positive colocalization) to black (negative colocalization), and background (colocalization score arbitrarily set to 0 ) in green. Image size: $6146 \times 6236$ in (B), 6152×6195 in (d). (e) Quantification of the percentage of colocalization for the indicated protein pairs. Data range from min to max. Dots correspond to individual cells issued from $n=3$ independent experiments. ${ }^{*} p<0.05$ 


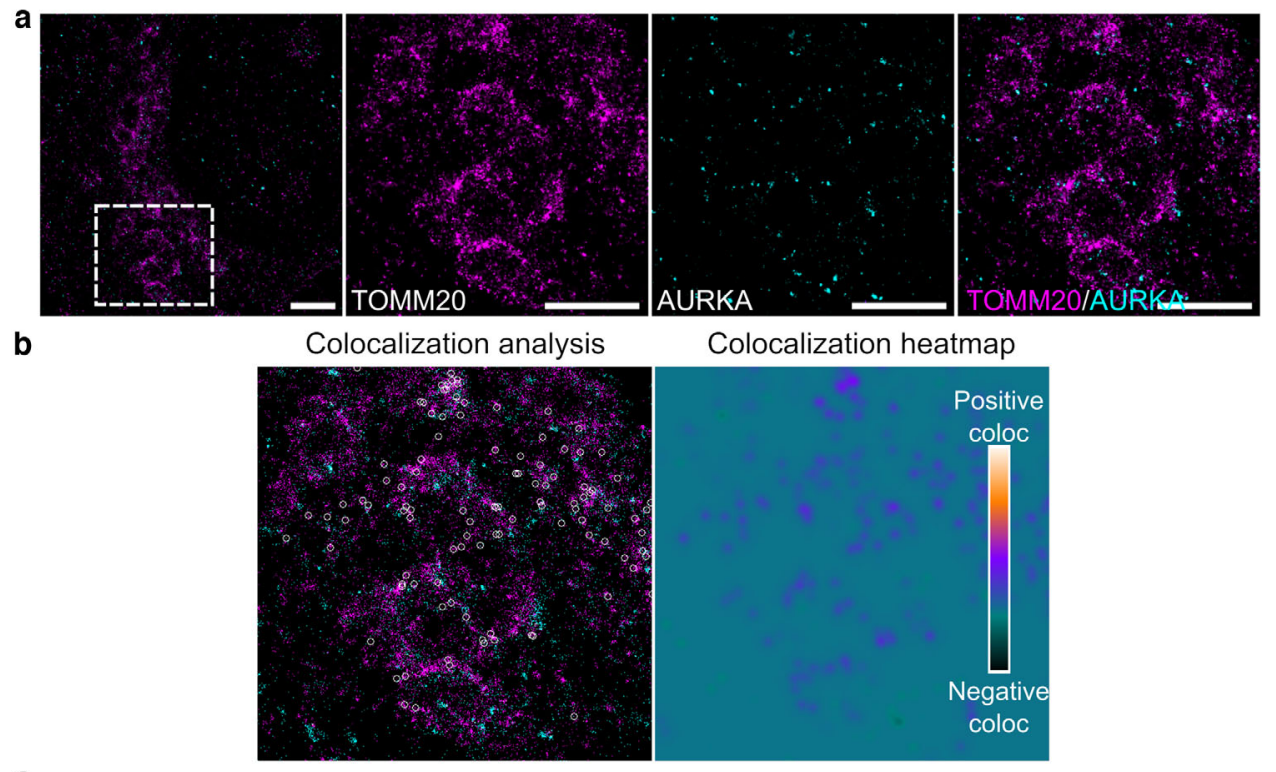

C
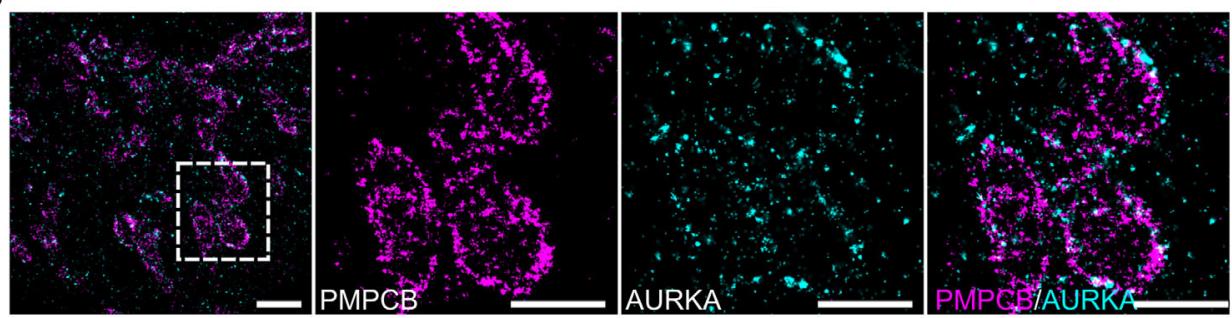

d

Colocalization analysis

Colocalization heatmap
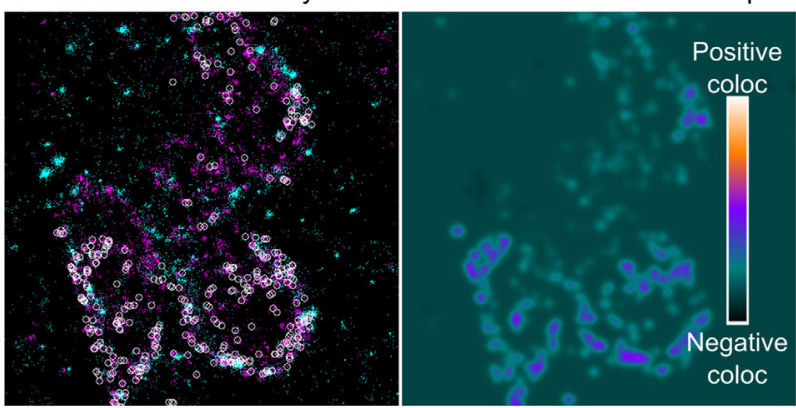

e

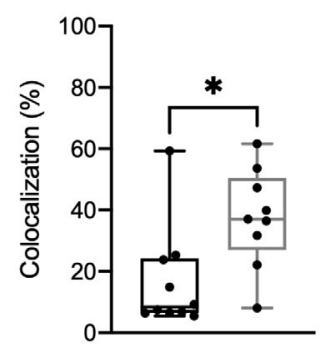

AURKATTOMM20

AURKAIPMPCB

FIGURE 4 AURKA mainly colocalizes with PMPCB in methanol-fixed cells. (a-c) Maximal projections of representative 2D dSTORM micrographs from MCF7 co-stained for AURKA and TOMM20 (a), or for AURKA and PMPCB (c). The anti-TOMM20 and anti-PMPCB primary antibodies were detected using secondary antibodies conjugated to Alexa 647 and pseudocolored magenta, while the anti-AURKA primary antibody was detected using a secondary antibody conjugated to Alexa 555 and pseudocolored cyan. For both protein pairs, the dotted area in the left panels indicates the magnified region where each staining is shown individually (middle panels), or merged (right panels). Scale bar: 2 $\mu \mathrm{m}$ (left) or $1 \mu \mathrm{m}$ (middle and right panels). (b-d) (Left panels) Visual representation of positive (white rounds) colocalization sites on the magnified area of AURKA/TOMM20 (b) and of AURKA/PMPCB (d) micrographs. (Right panels) Colocalization heatmap ranging from yellow (positive colocalization) to black (negative colocalization), and background (colocalization score arbitrarily set to 0 ) in green. Image size: $3522 \times 3524$ in (b), 2359×2259 in (d). (e) Quantification of the percentage of colocalization for the indicated protein pairs. Data range from min to max. Dots correspond to individual cells issued from $n=3$ independent experiments. ${ }^{*} p<0.05$ 
a

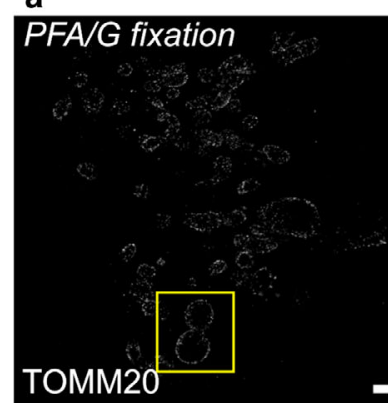

Methanol fixation

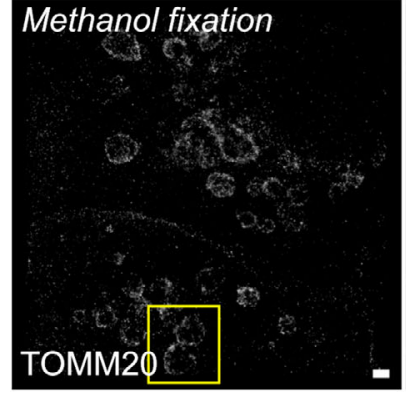

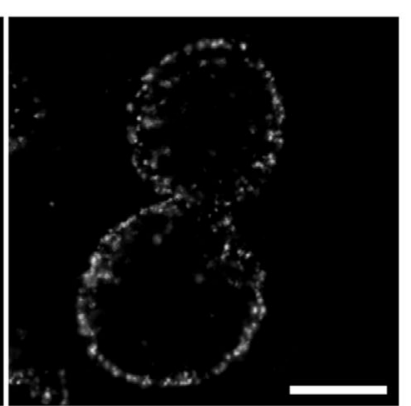

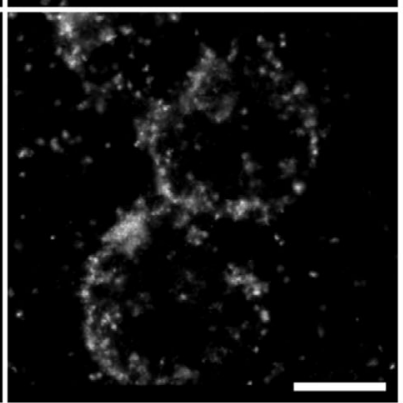

b

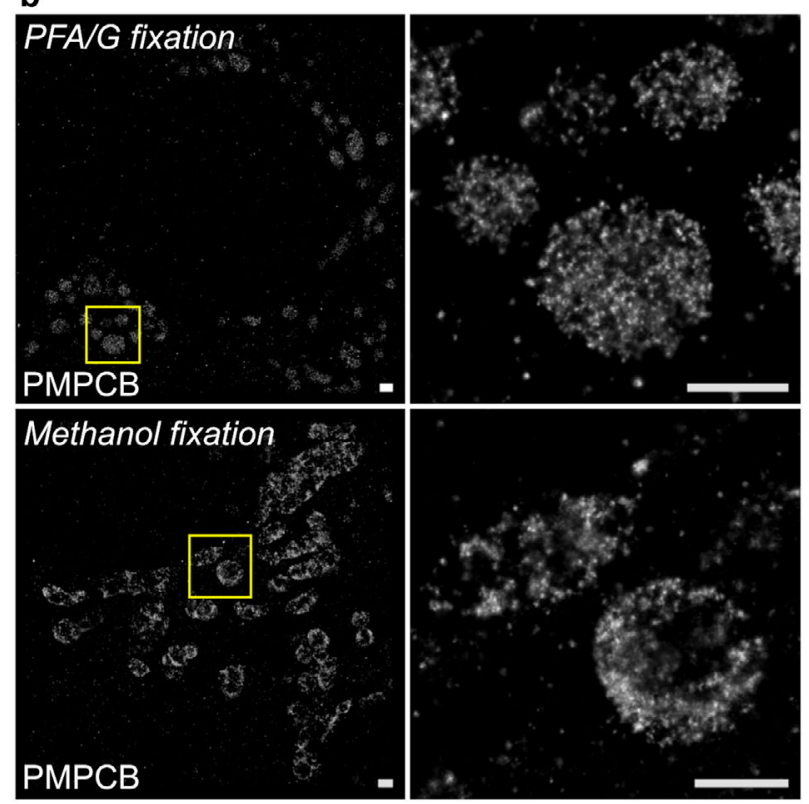

FIGURE 5 Methanol fixation degrades mitochondrial ultrastructure. (a-b) Representative images of MCF7 cells stained for TOMM20 (a) or PMPCB (b) after being fixed in a PFA/G mixture (upper panels), or in ice-cold methanol (lower panels). Primary antibodies were detected using secondary antibodies conjugated to Alexa 647. The yellow insets indicate the magnified region for each protein. Scale bar: $1 \mu \mathrm{m}$

and of the AURKA/PMPCB pairs were similar, both of nearly $\sim 16 \%$ (Figure $6(\mathrm{~b}, \mathrm{~d}, \mathrm{e})$ ). While colocalization values for the AURKA/TOMM20 pair were similar in methanol- and PFA/G-fixed cells (Figures 4(e) and 6(e)), the AURKA/PMPCB colocalization coefficients were significantly lowered in PFA/G-fixed cells (14.3\%) compared to those obtained in methanol-fixed cells $(38 \%)$ (Figures 4(e) and (6e)). Although PFA/G fixation globally ameliorated mitochondrial ultrastructure, we did not retrieve the preferential colocalization of AURKA with PMPCB previously observed in methanol-fixed cells (Figure 4(e)).

In conclusion, PFA/G fixation coupled to antigen retrieval procedures is less sensitive than a methanolbased fixation towards the matrix pool of AURKA. Therefore, an alcohol-based fixation should be preferred to detect the proximity of AURKA with matrix proteins, although it partially compromises the integrity of mitochondrial subcompartments. However, the quantity of OMM-bound AURKA detected using a PFA/G fixation is similar to that obtained with a methanol-based fixation. Therefore, PFA/G fixation appears to be a convenient approach to explore the functional relevance of the OMM pool of AURKA with greater specificity, and this while preserving mitochondrial morphology.

\section{DISCUSSION}

As a proof of concept of the methodology, our data show that dSTORM coupled to GcoPS qualitatively and quan- titatively separates the different mitochondrial subcompartments. In addition, with this approach we showed that dSTORM is sensitive enough to spatially resolve the localization of integral mitochondrial proteins on individual mitochondrial subcompartments, such as the OMM or the IMM/matrix. This confirms a recent elegant report, where two-color dSTORM and MINFLUX were used to follow the distribution of MICOS subunits throughout mitochondrial cristae (Stephan et al., 2020).

We also show for the first time that dSTORM is suitable to detect non-integral mitochondrial proteins as AURKA, which have multiple subcellular locations and are only transiently present at this compartment. This indeed confirms that dSTORM provides an exquisite spatial resolution, but it also indicates that sample preparation procedures must be carefully optimized to preserve subcellular structures while detecting the protein(s) of interest, or their specific sub-organellar pools. Despite these great advantages, it should also be noted that the temporal resolution of dSTORM is limited, mostly due to the composition of blinking buffers and imaging conditions which are largely incompatible with living samples. In this light, the recent development of MINFLUX appears to be a very promising alternative, combining a high spatial resolution with the possibility of using living samples (Balzarotti et al., 2017).

In terms of investigating mitochondrial functions, dSTORM is a useful method to define the submitochondrial locations of proteins with unprecedented resolution. The MitoCarta repository-the largest public database annotating mitochondrial proteins (Calvo et al., 2016) - 

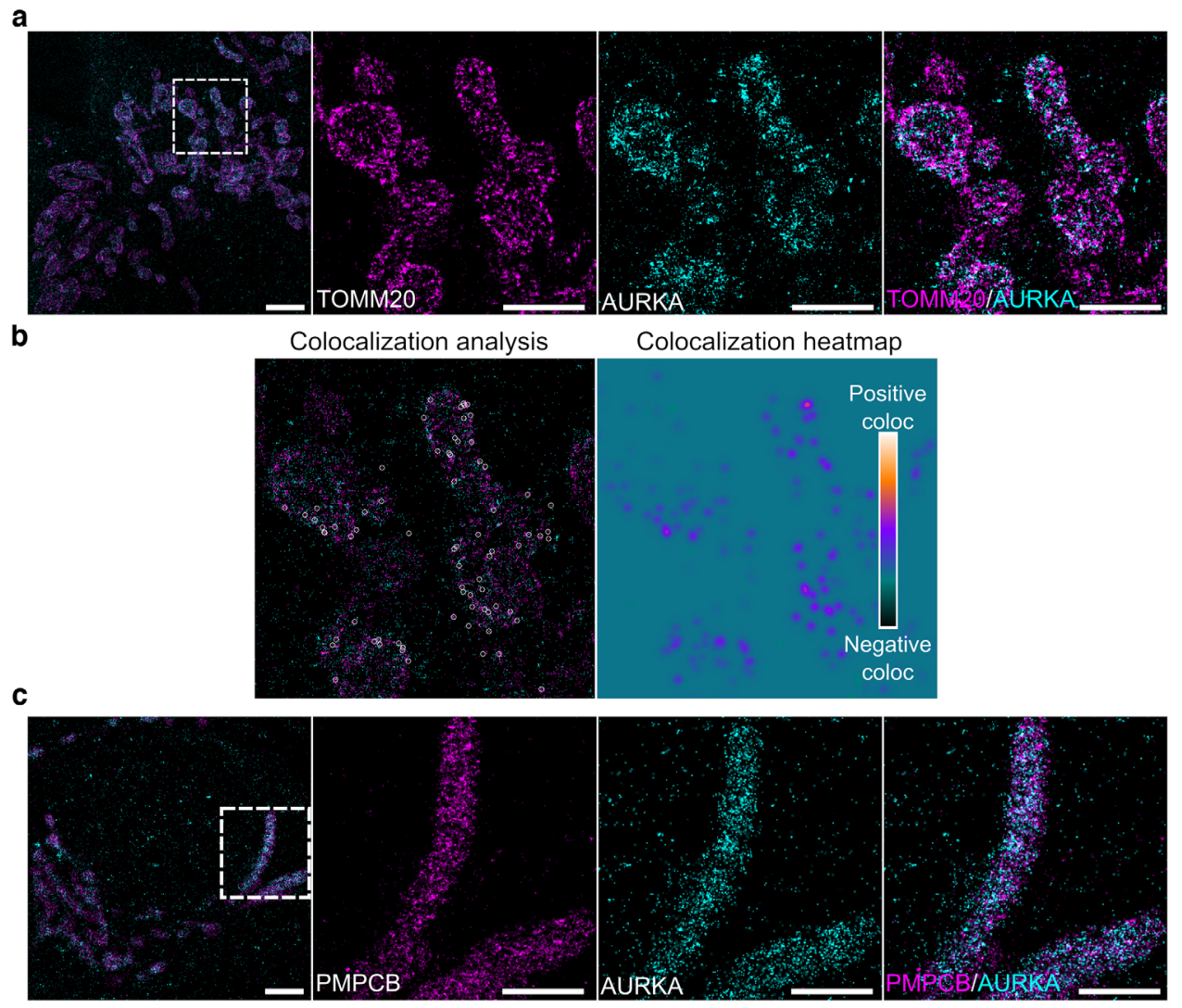

d

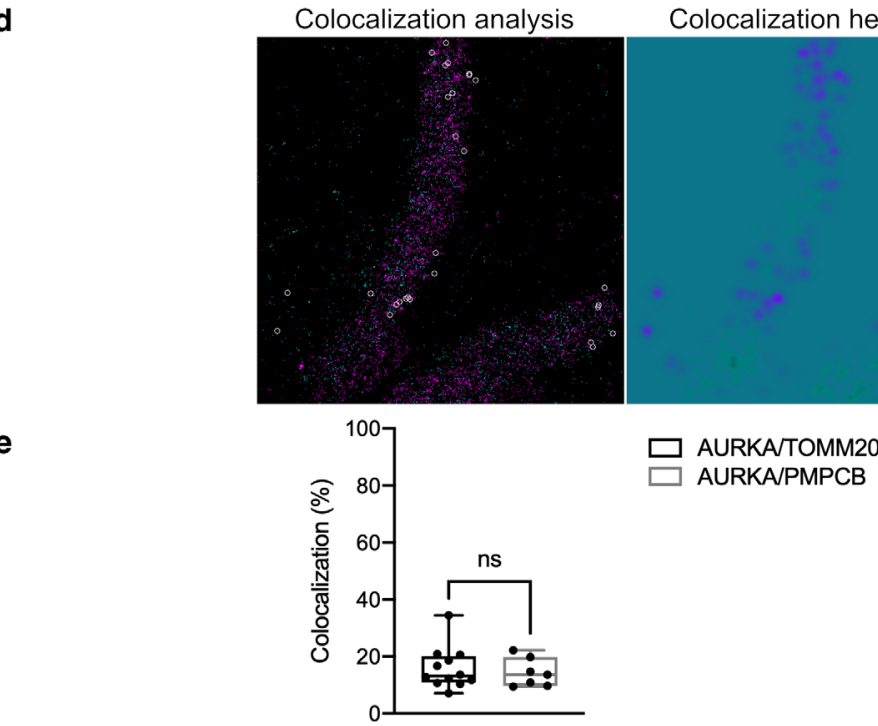

Colocalization heatmap

FIGURE 6 PFA/G fixation coupled to antigen retrieval procedures are more specific towards the OMM pool of AURKA. (a-c) Maximal projections of representative 2D dSTORM micrographs from MCF7 co-stained for AURKA and TOMM20 (a), or for AURKA and PMPCB (b). The anti-TOMM20 and anti-PMPCB primary antibodies were detected using secondary antibodies conjugated to Alexa 647 and pseudocolored magenta, while the anti-AURKA primary antibody was detected using a secondary antibody conjugated to Alexa 555 and pseudocolored cyan. For both protein pairs, the dotted area in the left panels indicates the magnified region where each staining is shown individually (middle panels), or merged (right panels). Scale bar: $2 \mu \mathrm{m}$ (left) or $1 \mu \mathrm{m}$ (middle and right panels). (b-d) (Left panels) Visual representation of positive (white rounds) colocalization sites on the magnified area of AURKA/TOMM20 (b) and of AURKA/PMPCB (d) micrographs. (Right panels) Colocalization heatmap ranging from yellow (positive colocalization) to black (negative colocalization) and background (colocalization score arbitrarily set to 0 ) in green. Image size: $6283 \times 6243$ in (b), $4859 \times 4894$ in (d). (e) Quantification of the percentage of colocalization for the indicated protein pairs. Data range from min to max. Dots correspond to individual cells issued from $n=3$ independent experiments. ns, not significant 
was recently found to contain only half of the mitochondrial proteome (Antonicka et al., 2020). Bio-ID-based proteomics techniques significantly expanded our view of the mitochondrial proteome (Antonicka et al., 2020), including ER-mitochondrial contacts (Cho et al., 2020; Kwak et al., 2020). However, they lack in showing the distribution of proteins within the organelles, which could be helpful in inferring their function(s) at this compartment. In particular, this would represent a huge advantage for proteins that are still poorly characterized from the functional point of view. The orthogonal validation of selected hits-obtained with large-scale omics-with in cellulo dSTORM could represent a new frontier to infer on protein functions in specific submitochondrial compartments.

Last, the use of dSTORM provides new perspectives on the roles played by mitochondrial AURKA. Not only it allows to observe the localization of the kinase within single mitochondria, but it also allows to evaluate its physical proximity with new/potential substrates. This would undoubtedly provide researchers with an unprecedented insight on the multiple roles of AURKA at mitochondria. However, it should be kept in mind that the proximity of AURKA with potential substrates or interactors should also be corroborated with complementary information on the activation status of the kinase. AURKA undergoes activation upon autophosphorylation on Thr288 (Bayliss et al., 2003; Cheetham, 2002; Zhang et al., 2007) before phosphorylating its substrates, both at mitosis and during interphase (reviewed in (Bertolin and Tramier, 2019; Nikonova et al., 2013)). A Förster's Resonance Energy Transfer (FRET) biosensor monitoring activated AURKA in single cells revealed that AURKA is activated in mitochondria (Bertolin et al., 2016). A fascinating future challenge could be to simultaneously monitor the activation of AURKA and its proximity with a putative substrate not in single cells, but in single mitochondria. Coupling FRET with dSTORM or, more generally, super-resolution microscopy could pave the way to the establishment of the mitochondrial "activome" of AURKA. In a therapeutic perspective, this functional network could be a promising tool for the treatment of patients with epithelial or hematological malignancies linked to the overexpression of AURKA.

\section{MATERIALS AND METHODS}

\section{Cell culture, fixation and antigen unmasking procedures}

Mycoplasma-free MCF7 cells (HTB-22) were purchased from the American Type Culture Collection (ATCC) and grown in Dulbecco's modified Eagle's medium (DMEM, Life Technologies Thermo Fisher Scientific), supplemented with 10\% Fetal Bovine Serum (FBS, Life
Technologies Thermo Fisher Scientific) and 1\% penicillin/streptomycin (Life Technologies Thermo Fisher Scientific) were cultured in 6-well plates on $1.5 \mathrm{H}$ 22×22 mm coverslips (Marienfeld VWR ref: 630-2186), and then fixed in a mixture of methanol-free $4 \%$ paraformaldehyde (PFA, Euromedex EM-15710) and $0.2 \%$ Glutaraldehyde (Euromedex, EM-16221) in PBS $1 \mathrm{X}$ (Merck, P4417-100TAB), at $37^{\circ} \mathrm{C}$ for $20 \mathrm{~min}$, or with ice-cold methanol at $-20^{\circ} \mathrm{C}$ for $20 \mathrm{~min}$. The autofluorescence generated by Glutaraldehyde was neutralized by using $1 \mathrm{mg} / \mathrm{ml}$ sodium borohydride at room temperature for 10 min (Sigma-Aldrich, 452882). AURKA antigen retrieval was performed by incubating cells previously fixed in 4\% PFA $/ 0.2 \%$ Glutaraldehyde in a $10 \mathrm{mM}$ sodium citrate tribasic dihydrate solution (Sigma-Aldrich, C8532), $\mathrm{pH} 6$ at $98^{\circ} \mathrm{C}$ on a hot plate for $15 \mathrm{~min}$. To avoid evaporation, approximately $5 \mathrm{ml} /$ well of this solution were used. After letting the plate cool down for 20 min at room temperature, the cells were washed twice in PBS 1X with gentle rocking, then permeabilized with 0.1\% Triton X-100 (Sigma, $93443-100 \mathrm{ml}$ ) for $10 \mathrm{~min}$. Cells were then saturated with $5 \%$ Bovine Serum Albumin (BSA) (Merck, A4503 50G) in PBS 1X for $1 \mathrm{~h}$, with gentle rocking.

\section{Immunocytochemistry procedures}

Primary antibodies were as follows: monoclonal mouse anti-AURKA (Clone 5C3; (Cremet et al., 2003) at 1:20, anti-TOMM22 (Abcam, ab10436) at 1:5000, and anti-ATP5B (Santa Cruz Biotechnologies, sc-166462) at 1:500; monoclonal rabbit anti-TOMM20 (Abcam, ab186734) at 1:500; polyclonal rabbit anti-PMPCB (Proteintech, 16064-1-AP) at 1:400, anti-VDAC1 (Abcam, ab15895) at 1:1000 and used after antigen retrieval procedures, and anti-COX2 (Agier et al., 2012) at 1:2000. All primary antibodies were diluted in 5\% PBS/BSA and incubated overnight at $4{ }^{\circ} \mathrm{C}$. After 3 washes in $5 \%$ PBS/BSA for 10 min each with gentle rocking, AURKA, VDAC1 or COX2 were revealed by a species-specific Fab' secondary antibody coupled to Alexa 555 (Thermo Fisher Scientific, A-21430 or A-21425). TOMM22 was revealed with a species-specific Fab' secondary antibody coupled to Alexa 647 (Thermo Fisher Scientific, A-21237 or A-21246). TOMM20 and PMPCB were revealed with either secondary antibody depending on the co-stained protein. Both secondaries were used at a concentration of $1: 1000$ in 5\% PBS/BSA and incubated in for $1 \mathrm{~h}$ at $37^{\circ} \mathrm{C}$. The cells were then washed three times for $10 \mathrm{~min}$ in 1X PBS, then post-fixed in 4\% PFA (Merck, 8187081000) at room temperature for $5 \mathrm{~min}$, washed three times in 1X PBS and incubated for $5 \mathrm{~min}$ in $50 \mathrm{mM}$ ammonium chloride (Sigma-Aldrich, 254134) before mounting on cavity blades (Marienfeld, VWR, 630-1611), replenished with dSTORM buffer (Smart Kit, 
Abbelight) and sealed with dental paste (Rotec, Picodent twinsil speed 22, Ref: 13001002) to avoid oxygen exposure, immediately prior to observation.

\section{Image acquisition and reconstruction procedures}

Two-color dSTORM acquisitions were performed on an Abbelight super resolution microscope (Abbelight, France) constituted by an Olympus IX83 microscope, an Abbelight SAFe 180 nanoscopy module, two Oxxius lasers at 640 and at $532 \mathrm{~nm}$ (Oxxius, France), a pco.panda sCMOS camera (PCO AG, Germany), equipped with a 100X oil-immersion objective (NA 1.5), and driven by the NEO software (Abbelight, France). Particle quantification and the reconstruction of the super resolution images was performed with the NEO software, using the Gaussian fitting algorithm integrated within. To evaluate the effect of the fixation method on mitochondrial integrity, TOMM20 or PMPCB-specific images were acquired with a home-made dSTORM microscope, composed of a Nikon TIRF system, a $640 \mathrm{~nm}$ laser (Toptica, Germany), an EMCCD camera (Andor, UK) and controlled by the NIS software. A 100X objective (NA 1.49) and a 1.5X lens were used to acquire images. Images were reconstructed using the UNLOC plugin for the ImageJ software (ImageJ, NHI), (Mailfert et al., 2018). 30000 frames of approximately $250 \times 250$ pixels were analyzed with the parameter-free UNLOC [UNsupervised particule LOCalization algorithm v1.0] algorithm in the high-density mode, allowing to fit with a multiple emitter fitting model, to correct the local and temporal variations of the background. The $x y$ drift was corrected using a robust correlation method, and data were filtered according to these parameters (No filters parameters were modified except SNR Min $=20$ ). Finally, an Integrated-Gaussian mode was applied to render the reconstructed images with a sub-pixel zoom factor of 8, leading to a final pixel size of $107 \mathrm{~nm}$.

\section{GcoPS-based colocalization analyses}

The colocalization method takes as inputs two binary images corresponding to dSTORM super-localizations translated into $0-1$ images with a spatial resolution of 10 $\mathrm{nm}$. The molecules with a localization uncertainty higher than $15 \mathrm{~nm}$ were discarded a priori. Colocalization was then performed on small squared windows as follows: the colocalization score is derived by approximating the interaction strength between the two proteins by the area of the intersection between the two binary images. In (Lavancier et al., 2020), this score is normalized with respect to the variance and is proved to follow a standard normal distribution. Therefore, the two proteins are expected to colocalize in the window of interest if the $p$ -
TABLE 1 Number of GCoPS tests performedfor dSTORM images of key protein/protein vicinities

\begin{tabular}{|c|c|c|}
\hline Number of tests & Calculation time & Colocalization (\%) \\
\hline \multicolumn{3}{|l|}{ TOM22/COX2 } \\
\hline 500 tests & $15 s$ & 31.66 \\
\hline 1000 tests & $26 s$ & 31.43 \\
\hline 2500 tests & $58 s$ & 31.93 \\
\hline 5000 tests & $1 \min 56 s$ & 32.13 \\
\hline 1000 tests & $4 \min 8 s$ & 32.41 \\
\hline \multicolumn{3}{|l|}{ TOM22/PMPCB } \\
\hline 500 tests & $13 s$ & 71.29 \\
\hline 1000 tests & $24 s$ & 70.01 \\
\hline 2500 tests & $59 \mathrm{~s}$ & 69.96 \\
\hline 5000 tests & $1 \mathrm{~min} 50 \mathrm{~s}$ & 69.7 \\
\hline 10,000 tests & $3 \min 37 s$ & 69.11 \\
\hline \multicolumn{3}{|l|}{ AURKA/TOMM20 } \\
\hline 500 tests & $13 s$ & 24.6 \\
\hline 1000 tests & $24 s$ & 24.8 \\
\hline 2500 tests & $59 \mathrm{~s}$ & 23.32 \\
\hline 5000 tests & $1 \min 58 s$ & 23.83 \\
\hline 10,000 tests & $3 \min 42 \mathrm{~s}$ & 24.72 \\
\hline \multicolumn{3}{|l|}{ AURKA/PMPCB } \\
\hline 500 tests & $12 \mathrm{~s}$ & 53.31 \\
\hline 1000 test & $23 \mathrm{~s}$ & 55.13 \\
\hline 2500 tests & $56 \mathrm{~s}$ & 53.29 \\
\hline 5000 tests & $1 \min 55 s$ & 53.59 \\
\hline 10,000 tests & $3 \min 33 s$ & 53.3 \\
\hline \multicolumn{3}{|c|}{ AURKA/TOMM20 antigen retrieval } \\
\hline 500 tests & $21 \mathrm{~s}$ & 19.4 \\
\hline 1000 tests & $33 \mathrm{~s}$ & 19.74 \\
\hline 2500 tests & $1 \min 6 s$ & 20.47 \\
\hline 5000 tests & $2 \min 5 s$ & 20.86 \\
\hline 10,000 tests & $4 \min 4 s$ & 20.4 \\
\hline \multicolumn{3}{|c|}{ AURKA/PMPCB antigen retrieval } \\
\hline 500 tests & $18 s$ & 14.63 \\
\hline 1000 tests & $29 \mathrm{~s}$ & 12.79 \\
\hline 2500 tests & $1 \min 9 \mathrm{~s}$ & 13.09 \\
\hline 5000 tests & $1 \min 57 s$ & 13.65 \\
\hline 10,000 tests & $3 \min 42 \mathrm{~s}$ & 14.07 \\
\hline
\end{tabular}

value is lower than the significance level $\alpha(\alpha=0.05)$. This procedure is then repeated $N$ times by applying the colocalization test independently on square windows randomly drawn in the whole image. The size of patches is uniformly drawn in the ranges $64 \times 64$ to $128 \times 128$ pixels on dSTORM images. Last, a global colocalization score is defined as the ratio of positive responses in windows over the total number $N$ of tested windows. We considered $N=5000$ tests for dSTORM images. This value was chosen in a range between 500 and 10,000 


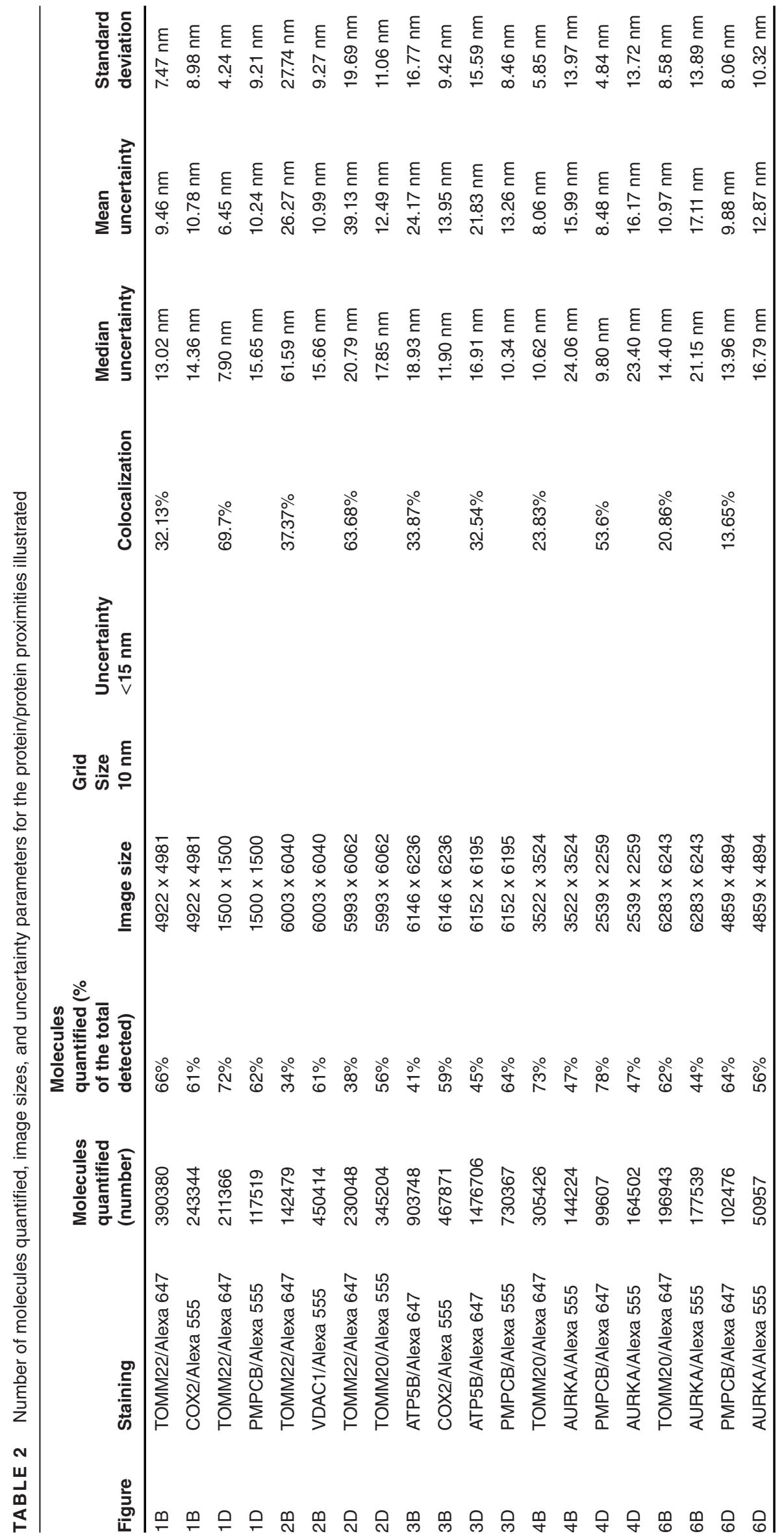


tests, for optimized calculation time and colocalization percentages (Table 1). The number of particles used for quantification and their percentages relative to the total number of particles retrieved in each image is reported in Table 2, together with the size of every image. For visualization purposes, the heatmaps were obtained by interpolating the score values using a Gaussian filter with a standard deviation of $50 \mathrm{~nm}$. Colocalization analyses were performed in $2 \mathrm{D}$, as the lateral and axial dimensions of organelles in cell lines ensure the analysis of individual mitochondria within one observation volume, and the signal/noise ratio is optimized on a larger number of particles than the few ones found on individual $z$ slices. The GcoPS analysis method is available on the Icy image analysis platform (http://icy.bioimageanalysis. org/plugin/gcops/).

\section{Statistical analyses}

After testing data for normality, the Kolmogorov-Smirnov test (Figures 1(e), 2(e), 3(e), 4(e), and 6(e)) was used to compare GcoPS colocalization scores in each condition.

\section{ACKNOWLEDGMENTS}

The authors thank M. Tramier, X. Pinson and R. F. Laine for their helpful comments and feedback on the present manuscript. The authors thank the Abbelight company for providing access to their acquisition and quantification systems prior to commercialization. Abbelight was not involved in experimental design, data handling, nor in the writing of the manuscript. The authors also wish to thank the microscopy platform of the Cochin Institute (IMAG'IC), Paris, France for preliminary experiments. The authors thank S. Dutertre at the Microscopy Rennes Imaging Center (MRic, Biologie, Santé, Innovation Technologique - BIOSIT, Rennes, France) for assistance, M. Garfa and all the colleagues at the Cell Imaging Platform, Structure Fédérative de Recherche Necker (Paris, France) for their support. MRic is member of the national infrastructure France-Biolmaging supported by the French National Research Agency (ANR10-INBS-04). This work was supported by the Centre National de la Recherche Scientifique (CNRS), an intramural BIOSIT grant, grants from the Ligue Contre le Cancer Comités d'llle et Vilaine, des Côtes d'Armor et $d u$ Finistère, the Fondation ARC pour la Recherche sur le Cancer, and an intramural technology-transfer grant from the Réseau Technologique Microscopie photonique de Fluorescence Multidimensionnelle (RTmfm) to G.B.

\section{CONFLICT OF INTEREST}

The authors declare no conflict of interest.

\section{AUTHOR CONTRIBUTIONS}

Béatrice Durel developed and optimized mitochondriaand AURKA-specific staining procedures, performed the experiments and particle quantification and reconstruction; Charles Kervrann optimized the GcoPS software for mitochondrial images, performed colocalization analyses and reviewed the manuscript; Giulia Bertolin designed research, provided funding and wrote and revised the manuscript. All the authors provided feedback on the manuscript and agreed on its final version.

\section{ORCID}

Giulia Bertolin (1) https://orcid.org/0000-0002-7359-

5733

\section{REFERENCES}

Agier, V., Oliviero, P., Lainé, J., L'Hermitte-Stead, C., Girard, S., Fillaut, S., Jardel, C., Bouillaud, F., Bulteau, A.L. \& Lombès, A. (2012) Defective mitochondrial fusion, altered respiratory function, and distorted cristae structure in skin fibroblasts with heterozygous OPA1 mutations. Biochimica et Biophysica Acta - Molecular Basis of Disease, 1822, 1570-1580.

Antonicka, H., Lin, Z.-Y., Janer, A., Aaltonen, M.J., Weraarpachai, W., Gingras, A.-C. \& Shoubridge, E.A. (2020) A high-density human mitochondrial proximity interaction network. Cell Metabolism, 32, 479-497.e9.

Balzarotti, F., Eilers, Y., Gwosch, K.C., Gynnå, A.H., Westphal, V., Stefani, F.D., Elf, J. \& Hell, S.W. (2017) Nanometer resolution imaging and tracking of fluorescent molecules with minimal photon fluxes. Science, 355, 606-612.

Bayliss, R., Sardon, T., Vernos, I. \& Conti, E. (2003) Structural basis of Aurora-A activation by TPX2 at the mitotic spindle. Molecular Cell, 12, 851-862.

Bertolin, G. \& Tramier, M. (2020) Insights into the non-mitotic functions of Aurora kinase A: more than just cell division. Cellular and Molecular Life Sciences, 77(6), 1031-1047.

Bertolin, G., Sizaire, F., Herbomel, G., Reboutier, D., Prigent, C. \& Tramier, M. (2016) A FRET biosensor reveals spatiotemporal activation and functions of aurora kinase A in living cells. Nature Communications, 7, 12674.

Bertolin, G., Bulteau, A.-L., Alves-Guerra, M.-C., Burel, A., Lavault, M.T., Gavard, O., Le Bras, S., Gagné, J.-P., Poirier, G.G., Le Borgne, R., Prigent, C., \& Tramier, M. (2018) Aurora kinase A localises to mitochondria to control organelle dynamics and energy production. ELife, 7, e38111.

Bolte, S. \& Cordelieres, F.P. (2006) A guided tour into subcellular colocalization analysis in light microscopy. Journal of Microscopy, 224, 213-232.

Boykins, L.G., Jones, J.C.R., Estraño, C.E., Schwartzbach, S.D. \& Skalli, O. (2016) Pre-embedding double-label immunoelectron microscopy of chemically fixed tissue culture cells. In HighResolution Imaging of Cellular Proteins, S.D. Schwartzbach, O. Skalli, and T. Schikorski, eds. (New York, NY: Springer New York), pp. 217-232.

Bozgeyik, E., Arman, K. \& Igci, Y.Z. (2015) Review: ATP5B (ATP synthase, $\mathrm{H}+$ transporting, mitochondrial $\mathrm{F} 1$ complex, beta polypeptide) Atlas of Genetics and Cytogenetics in Oncology and Haematologyv,

Calvo, S.E., Clauser, K.R. \& Mootha, V.K. (2016) MitoCarta2.0: an updated inventory of mammalian mitochondrial proteins. Nucleic Acids Research, 44, D1251-1257.

Camara, A.K.S., Zhou, Y., Wen, P.-C., Tajkhorshid, E. \& Kwok, W.M. (2017) Mitochondrial VDAC1: a key gatekeeper as potential 
therapeutic target. Frontiers in Physiology, 8, 460. https://www. frontiersin.org/articles/10.3389/fphys.2017.00460/full

Cheetham, G.M.T. (2002) Crystal structure of Aurora-2, an oncogenic serine/threonine kinase. Journal of Biological Chemistry, 277, 42419-42422.

Cho, K.F., Branon, T.C., Rajeev, S., Svinkina, T., Udeshi, N.D., Thoudam, T., Kwak, C., Rhee, H.-W., Lee, I.-K., Carr, S.A., \& Ting, A.Y. (2020) Split-TurbolD enables contact-dependent proximity labeling in cells. Proceedings of the National Academy of Sciences, 117(22), 12143-12154.

Cremet, J.Y., Descamps, S., Vérite, F., Martin, A. \& Prigent, C. (2003) Preparation and characterization of a human aurora-A kinase monoclonal antibody. Molecular and Cellular Biochemistry, 243, 123131.

Damodaran, A.P., Vaufrey, L., Gavard, O. \& Prigent, C. (2017) Aurora A Kinase is a priority pharmaceutical target for the treatment of cancers. Trends in Pharmacological Sciences, 38, 687-700.

Daniel, P., Halada, P., Jelínek, M., Balušíková, K. \& Kovář, J. (2019) Differentially expressed mitochondrial proteins in human MCF7 breast cancer cells resistant to paclitaxel. International Journal of Molecular Sciences, 20, 2986.

Dlasková, A., Engstová, H., Špaček, T., Kahancová, A., Pavluch, V., Smolková, K., Špačková, J., Bartoš, M., Hlavatá, L.P. \& Ježek, P. (2018) 3D super-resolution microscopy reflects mitochondrial cristae alternations and mtDNA nucleoid size and distribution. Biochimica et Biophysica Acta (BBA) - Bioenergetics, 1859, 829844.

Enger, R. (2017) Automated gold particle quantification of immunogold labeled micrographs. Journal of Neuroscience Methods, 286, 3137.

Frey, T.G. \& Mannella, C.A. (2000) The internal structure of mitochondria. Trends in Biochemical Sciences, 25, 319-324.

Golic, I., Aleksic, M., Lazarevic, A., Bogdanovic, M., Jonic, S. \& Korac, A. (2016) Methods for studying the localization of mitochondrial complexes III and IV by immunofluorescent and immunogold microscopy. Archives of Biological Sciences, 68, 767-772.

Grant, R., Abdelbaki, A., Bertoldi, A., Gavilan, M.P., Mansfeld, J., Glover, D.M. \& Lindon, C. (2018) Constitutive regulation of mitochondrial morphology by Aurora A kinase depends on a predicted cryptic targeting sequence at the $\mathrm{N}$-terminus. Open Biology, 8 , 170272.

Hayat, M.A. (1992) Quantitation of immunogold labeling. Micron and Microscopica Acta, 23, 1-16.

Heilemann, M., van de Linde, S., Schüttpelz, M., Kasper, R., Seefeldt, B., Mukherjee, A., Tinnefeld, P. \& Sauer, M. (2008) Subdiffractionresolution fluorescence imaging with conventional fluorescent probes. Angewandte Chemie, International Edition, 47, 6172-6176.

Hoetelmans, R.W.M., Prins, F.A., Velde, I.C., van der Meer, J., van de Velde, C.J.H. \& van Dierendonck, J.H. (2001) Effects of acetone, methanol, or paraformaldehyde on cellular structure, visualized by reflection contrast microscopy and transmission and scanning electron microscopy. Applied Immunohistochemistry \& Molecular Morphology, 9, 346-351.

Huang, B., Jones, S.A., Brandenburg, B. \& Zhuang, X. (2008) Wholecell 3D STORM reveals interactions between cellular structures with nanometer-scale resolution. Nature Methods, 5, 1047-1052.

Jakobs, S. \& Wurm, C.A. (2014) Super-resolution microscopy of mitochondria. Current Opinion in Chemical Biology, 20, 9-15.

Jamur, M.C. \& Oliver, C. (2010) Permeabilization of Cell Membranes. In Immunocytochemical Methods and Protocols, C. Oliver, and M.C. Jamur, eds. (Totowa, NJ: Humana Press), pp. 63-66.

Kaasik, A., Safiulina, D., Zharkovsky, A. \& Veksler, V. (2007) Regulation of mitochondrial matrix volume. American Journal of PhysiologyCell Physiology, 292, C157-C163.

Kashatus, D.F., Lim, K.-H., Brady, D.C., Pershing, N.L.K., Cox, A.D. \& Counter, C.M. (2011) RALA and RALBP1 regulate mitochondrial fission at mitosis. Nature Cell Biology, 13, 1108-1115.
Kwak, C., Shin, S., Park, J.-S., Jung, M., Nhung, T.T.M., Kang, M.-G., Lee, C., Kwon, T.-H., Park, S.K., Mun, J.Y., Kim, J.-S., \& Rhee, H.W. (2020) Contact-ID, a tool for profiling organelle contact sites, reveals regulatory proteins of mitochondrial-associated membrane formation. Proceedings of the National Academy of Sciences, 201916584.

van der Laan, M., Horvath, S.E. \& Pfanner, N. (2016) Mitochondrial contact site and cristae organizing system. Current Opinion in Cell Biology, 41, 33-42.

Lavancier, F., Pécot, T., Zengzhen, L. \& Kervrann, C. (2019) Testing independence between two random sets for the analysis of colocalization in bioimaging. Biometrics, $n / a$.

Lavancier, F., Pécot, T., Zengzhen, L. \& Kervrann, C. (2020) Testing independence between two random sets for the analysis of colocalization in bioimaging. Biometrics, 76, 36-46.

Mailfert, S., Touvier, J., Benyoussef, L., Fabre, R., Rabaoui, A., Blache, M.-C., Hamon, Y., Brustlein, S., Monneret, S., Marguet, D. \& Bertaux, N. (2018) A theoretical high-density nanoscopy study leads to the design of UNLOC, a parameter-free algorithm. Biophysical Journal, 115, 565-576.

Melan, M.A. (1999) Overview of Cell Fixatives and Cell Membrane Permeants. In Immunocytochemical Methods and Protocols, (New Jersey: Humana Press), pp. 45-56.

Naso, F.D., Boi, D., Ascanelli, C., Pamfil, G., Lindon, C., Paiardini, A. \& Guarguaglini, G. (2021) Nuclear localisation of Aurora-A: its regulation and significance for Aurora-A functions in cancer. Oncogene, 40, 3917-3928.

Neumann, D., Bückers, J., Kastrup, L., Hell, S.W. \& Jakobs, S. (2010) Two-color STED microscopy reveals different degrees of colocalization between hexokinase-I and the three human VDAC isoforms. PMC Biophysics, 3, 4.

Nikonova, A.S., Astsaturov, I., Serebriiskii, I.G., Dunbrack, R.L. \& Golemis, E.A. (2013) Aurora A kinase (AURKA) in normal and pathological cell division. Cellular and Molecular Life Sciences, 70, 661-687.

Nunnari, J. \& Suomalainen, A. (2012) Mitochondria: in sickness and in health. Cell, 148, 1145-1159.

Reichert, A.S. \& Neupert, W. (2002) Contact sites between the outer and inner membrane of mitochondria-role in protein transport. Biochimica et Biophysica Acta - Molecular Cell Research, 1592, 41-49.

Samanta, S., Gong, W., Li, W., Sharma, A., Shim, I., Zhang, W., Das, P., Pan, W., Liu, L., Yang, Z., Qu, J. \& Kim, J.S. (2019) Organic fluorescent probes for stochastic optical reconstruction microscopy (STORM): Recent highlights and future possibilities. Coordination Chemistry Reviews, 380, 17-34.

Sarmiento-Salinas, F.L., Delgado-Magallón, A., Montes-Alvarado, J.B., Ramírez-Ramírez, D., Flores-Alonso, J.C., Cortés-Hernández, P., Reyes-Leyva, J., Herrera-Camacho, I., Anaya-Ruiz, M., Pelayo, R., Millán-Pérez-Peña, L. \& Maycotte, P. (2019) Breast cancer subtypes present a differential production of reactive oxygen species (ROS) and susceptibility to antioxidant treatment. Frontiers in Oncology, 9.

Schnell, U., Dijk, F., Sjollema, K.A. \& Giepmans, B.N.G. (2012) Immunolabeling artifacts and the need for live-cell imaging. Nature Methods, 9, 152-158.

Siegmund, S.E., Grassucci, R., Carter, S.D., Barca, E., Farino, Z.J., Juanola-Falgarona, M., Zhang, P., Tanji, K., Hirano, M., Schon, E.A., Frank, J. \& Freyberg, Z. (2018) Three-dimensional analysis of mitochondrial crista ultrastructure in a patient with leigh syndrome by in situ cryoelectron tomography. IScience, 6, 83-91.

Stephan, T., Brüser, C., Deckers, M., Steyer, A.M., Balzarotti, F., Barbot, M., Behr, T.S., Heim, G., Hübner, W., Ilgen, P., Lange, F., PacheuGrau, D., Pape, J.K., Stoldt, S., Huser, T., Hell, S.W., Möbius, W., Rehling, P., Riedel, D. \& Jakobs, S. (2020) MICOS assembly controls mitochondrial inner membrane remodeling and crista junction redistribution to mediate cristae formation. EMBO Journal, 39(14), e104105. 
Vincent, A.E., Ng, Y.S., White, K., Davey, T., Mannella, C., Falkous, G., Feeney, C., Schaefer, A.M., McFarland, R., Gorman, G.S., TayIor, R.W., Turnbull, D.M. \& Picard, M. (2016) The spectrum of mitochondrial ultrastructural defects in mitochondrial myopathy. Scientific Reports, 6, 30610.

Vogel, F., Bornhövd, C., Neupert, W. \& Reichert, A.S. (2006) Dynamic subcompartmentalization of the mitochondrial inner membrane. Journal of Cell Biology, 175, 237-247.

van Wilpe, S., Ryan, M.T., Hill, K., Maarse, A.C., Meisinger, C., Brix, J., Dekker, P.J., Moczko, M., Wagner, R., Meijer, M., Guiard, B., Hönlinger, A. \& Pfanner, N. (1999) Tom22 is a multifunctional organizer of the mitochondrial preprotein translocase. Nature, 401, 485-489.
Zhang, Y., Ni, J., Huang, Q., Ren, W., Yu, L. \& Zhao, S. (2007) Identification of the auto-inhibitory domains of Aurora-A kinase. Biochemical and Biophysical Research Communications, 357, 347-352.

How to cite this article: Durel, B., Kervrann, C. \& Bertolin, G. (2021) Quantitative dSTORM super-resolution microscopy localizes Aurora kinase A/AURKA in the mitochondrial matrix. Biology of the Cell, 1-16.

https://doi.org/10.1111/boc.202100021 